\title{
Macrophages in Giemsa-stained cerebrospinal fluid specimens predict carcinomatous meningitis
}

\author{
SAYAKA KOBAYASHI ${ }^{1}$, MASANAO SAIO $^{1}$, MISA FUJIMORI $^{1}$, JUNKO HIRATO $^{2,3}$, \\ TETSUNARI OYAMA ${ }^{2,4}$ and TOSHIO FUKUDA ${ }^{1}$ \\ ${ }^{1}$ Laboratory of Histopathology and Cytopathology, Department of Laboratory Sciences, \\ Gunma University Graduate School of Health Sciences, Maebashi, Gunma 371-8514; \\ ${ }^{2}$ Clinical Department of Pathology, Gunma University Hospital, Maebashi, Gunma 371-8511; \\ ${ }^{3}$ Department of Pathology, Public Tomioka General Hospital, Tomioka, Gunma, 370-2316; ${ }^{4}$ Department of \\ Diagnostic Pathology, Gunma University Graduate School of Medicine, Maebashi, Gunma 371-8511, Japan
}

Received March 16, 2020; Accepted September 15, 2020

DOI: $10.3892 / \mathrm{ol} .2020 .12217$

\begin{abstract}
Carcinomatous meningitis is a condition in which tumor cells spread to the subarachnoid space. Leukocyte counting and typing of cerebrospinal fluid (CSF) cell components are performed manually or using flow cytometry. However, a detailed analysis of these variables using cytological specimens has not yet been reported. The present study analyzed cytological specimens using Giemsa staining and whole slide imaging with computer-assisted image analysis (CAIA) to clarify the characteristics of the leukocyte population in CSF, especially in carcinomatous meningitis. Manual evaluation was performed using 280 Giemsa-stained cytological CSF specimens. For 49 samples, CAIA was used for the whole area of Papanicolaou (Pap) staining, and Giemsa-stained specimens of the same samples were imaged using a virtual slide scanner. The nuclear morphology of the leukocytes was assessed, and the total leukocyte and leukocyte subset (lymphocytes, neutrophils and macrophages) counts were evaluated. Then, the number and percentage of each leukocyte subset population were evaluated. The total leukocyte count was significantly higher in Giemsa-stained specimens compared with in Pap-stained specimens. The percentage of macrophages was significantly higher in samples from patients with non-hematological tumors compared with in samples from patients without tumors, which was confirmed by manual evaluation of the specimens. In addition, the cut-off value of the percentage of macrophages that could discriminate
\end{abstract}

Correspondence to: Dr Masanao Saio, Laboratory of Histopathology and Cytopathology, Department of Laboratory Sciences, Gunma University Graduate School of Health Sciences, Shared Facilities Building, 39-22, 3-chome, Showa, Maebashi, Gunma 371-8514, Japan

E-mail: saio@gunma-u.ac.jp

Key words: cerebrospinal fluid cytology, leukocyte, whole slide imaging, computer-assisted image analysis, Giemsa staining between the tumor history negative cases and cytologically tumor positive cases was determined, revealing that a higher proportion of macrophages reflected the existence of atypical/ malignant epithelial tumor cells in CSF samples. Thus, atypical cell screening and analysis of the background characteristics of the leukocyte population should be the focus of cytological specimen screening, especially not to miss carcinomatous meningitis.

\section{Introduction}

Carcinomatous meningitis (CM) is a condition in which tumor cells spread to the subarachnoid space hematogenously, via direct invasion, or through neuroinvasion (1), and occurs in at least $5 \%$ of patients with disseminated cancer (2). CM is diagnosed via neurological examination, computed Tomography, Magnetic Resonance Imaging, cerebrospinal fluid (CSF) analysis (cell count, opening pressure, levels of protein, glucose and lactate) and CSF cytology (1).

Leukocyte counting and typing of CSF has previously been performed manually or using various types of flow cytometers $(3,4)$. Normally, in healthy individuals the manual leukocyte count is $<5 / \mu 1$ (4). While, in 1987, Hayward et al (5) described that $77 \%$ of malignant meningitis (without specifying tumor types) showed $>5$ cells $/ \mathrm{ml}$ manual leukocyte count (5). Subsequently, MacKenzie (6) reported that in malignant meningitis including carcinomas, primary central nervous system (CNS) tumors, and hematopoietic tumors, 22 cases out of 26 confirmed malignancy cases showed raised manual leukocyte counts. While Djukic et al (7) reported that 66 cases out of 132 confirmed malignancies in malignant meningitis showed increased manual leukocyte counts. In terms of leukocyte subsets, diverse results have been reported following analysis. For example, the mononuclear cell count is elevated in $~ 50 \%$ of patients with malignancy, and the polymorphonuclear cell count is increased in $20 \%$ of patients (8). while, the frequencies of lymphocytes and granulocytes are also increased, whereas those of monocytes are decreased (7).

Notably, leukocyte counting and typing are also performed using flow cytometry (9). For example, Illan et al (9) analyzed 
absolute cell count, tumor cell count, and inflammatory cell count in malignant lymphoma cases and leptomeningeal carcinomaosis (9). On the other hand, in terms of cytological specimens, the features of malignant cells are the main focus of CSF cytology. For example, Singh et al (10) summarized cytological characteristics of tumor appearance patterns for breast carcinoma, ovarian carcinoma, malignant melanoma among others (10). Ho et al (11) summarized cytomorphological characteristics of primary CNS tumors in the CSF. Rao et al (12) reported cytopathological characteristics of CSF for 15 patients with breast cancer. Bell (13) reported the pitfalls which could interfere with the diagnostic accuracy in CSF cytology Bigner (14) comprehensively summarized CSF cytology from the preparation method to the cytological findings of non-infectious condition, infectious conditions, hematopoietic tumors, metastatic carcinomas and primary CNS tumors. Cutler and Spertell (15) mentioned the condition of sample preparation in order to maintain morphology of cells in the CSF and achieve good cytological observation to diagnose malignancy. In terms of characteristics of leukocytes, the findings have been evaluated manually in cytological specimens. For example, lymphocytes and monocytes mixed with erythrocytes and fibrin are observed in the background of metastatic breast cancer specimens (12), neutrophils are identified in malignant specimens of the central nervous system and eosinophils have been reported in patients with Hodgkin's lymphoma (5). Moreover, reactive and inflammatory infiltrates have been reported in the background when only a few metastatic cancer cells are detected in CSF (14). In addition, none of these aforementioned studies evaluated the leukocyte count and type using cytological specimens via an objective method, such as computer-assisted image analysis (CAIA). Furthermore, daily practice of screening CSF cytological specimens usually focuses on the detection of atypical cells, without much consideration given to the leukocyte population (10-14). Therefore, it is possible that the importance of background leukocyte evaluation in cytology specimens may have be overlooked.

Since 2016, the combination of whole slide imaging (WSI) and CAIA has been used in the fields of pathology and cytology (16). Yamada et al (16) analyzed the size, shape and texture of nuclei for specimens of proliferative breast lesions using CAIA. The group described CAIA as a valuable tool for distinguishing atypical hyperplasia and ductal carcinoma in situ from typical ductal hyperplasia. Kosuge et al (17) revealed that the nuclear features of invasive urothelial carcinoma differed from those of low-grade papillary urothelial carcinoma as assessed using the combination of WSI and CAIA. Furthermore, Eyraud et al (18) utilized CAIA to analyze the immunohistochemical staining-positive area for indoleamine 2,3-dioxygenase or forkhead box protein 3 in a tissue microarray of colorectal cancer tissue samples. Our previous study analyzed the nuclear features of lung adenocarcinoma and found that some adenocarcinomas showed low expression level of inner nuclear membrane protein emerin (19), and the low expression group showed enlarged size and oval shape of the nucleus in comparison with the emerin expressing group (19). Overall, the aforementioned reports indicated that WSI and CAIA are crucial for evaluating pathological and cytological specimens. Thus, the present study evaluated CSF cytology specimens utilizing WSI and CAIA to clarify the features of leukocyte subsets.

\section{Materials and methods}

Sample selection. All CSF cytology samples, except ones of patients who declined participation for the study, collected at Gunma University Hospital (Maebashi, Japan) from January 2001 to December 2011 were reviewed using the hospital information system. Pediatric cases, cases with no description of the clinical diagnosis, unavailable cases and cases without a histological diagnosis were excluded. Finally, 339 samples were examined by manually observing Giemsa-stained specimens. For patients who underwent multiple cytological examinations of CSF, the specimen with malignant cytology that featured the highest number of cells was used for subsequent analysis. The remaining 280 samples were utilized for manual observation of the microscopically analyzed Giemsa specimens. Of the 280 patients included in the present study, 145 were male and 135 female. The patients had a median age, 61.0 years; mean age, 56.1 years and age range, $20-86$ years.

Ethics approval. The present study was approved by the Gunma University Ethical Review Board for Medical Research Involving Human Subjects of Gunma University School of Medicine (GUERB; Gunma, Japan), and the written notification for the current study was presented publicly on the webpage of Gunma University Hospital. Furthermore, the possibility to decline participation in this study was provided according to the Ethical Guidelines for Medical and Health Research Involving Human Subjects of the Japanese government (Ministry of Education, Culture, Sports, Science and Technology, and Ministry of Health, Labour and Welfare). Informed consent was waived by GUERB based on the above guidelines due to the retrospective nature of the study.

Manual cell number evaluation and specimen condition evaluation. Already prepared Giemsa-stained and Papanicolaou (Pap)-stained specimens which were formerly prepared in the Clinical Department of Pathology for daily practice were used. The staining protocol for Pap smear used in Gunma University Hospital is described below. All steps were done at room temperature. One drop of $30 \%$ albumin was added to the CSF samples. Silane-coated slide glass was assembled to Autosmear ${ }^{\mathrm{TM}}$ apparatus (Sakura Finetek Japan Co., Ltd.) and the apparatus was set to roter of Autosmear ${ }^{\mathrm{TM}}$ centrifuge with CSF samples. Then the samples were centrifuged for $5 \mathrm{~min}$ at $48 \mathrm{x} \mathrm{g}$. The supernatant was discarded and the slide glass was removed from the apparatus. The specimen was fixed in $95 \%$ ethanol for 15 min followed by $70 \%$ ethanol (30 sec), $50 \%$ ethanol $(30 \mathrm{sec})$ and running water (30 sec twice). Then the specimens were stained by Gil's hematoxylin solution for $1.5 \mathrm{~min}$ followed by $0.5 \%$ hydrochloric acid in $70 \%$ ethanol (30 sec), running water (5 min), $70 \%$ ethanol (30 sec), $80 \%$ ethanol (30 sec) and $95 \%$ ethanol (30 sec). Then the specimens were stained by OG-6 solution followed by $95 \%$ ethanol for $15 \mathrm{sec}$. Then the specimens were treated with $1 \%$ phosphotungstic acid solution $(30 \mathrm{sec})$ followed by $95 \%$ ethanol $(30 \mathrm{sec})$. Then the specimens were 
stained using EA-50 solution $5 \mathrm{~min}$ ) followed by $95 \%$ ethanol (30 sec), 95\% ethanol ( $1 \mathrm{~min}), 100 \%$ ethanol ( $1 \mathrm{~min}, 4$ times) and xylene (1 min, thrice). Finally the specimen's coverslipping was performed. For Giemsa staining, all steps were done at room temperature. One drop of $30 \%$ albumin was added to CSF sample. Silane-coated slide glass was assembled to Autosmear $^{\mathrm{TM}}$ apparatus (Sakura Finetek Japan Co., Ltd.,) and the apparatus was set to roter of Autosmear ${ }^{\mathrm{TM}}$ centrifuge with CSF sample. Then the samples were centrifuged for $5 \mathrm{~min}$ at $48 \mathrm{x}$ g. The supernatant was discarded and the slide glass was removed from the apparatus. The specimen was cold dried by air. Then the specimens were stained by May-Gruenwald solution for $2 \mathrm{~min}$ followed by running water for $30 \mathrm{sec}$. Then the specimens were stained by Giemsa solution for $15 \mathrm{~min}$ followed by running water for $40 \mathrm{sec}$. Then the specimens were completely cold dried by air. After rinsing in xylene for $1 \mathrm{~min}$, coverslipping of the specimens was performed. After evaluating the specimens using a light microscope (BX40; Olympus Corporation), a consensus was reached between a cytotechnologist (SK) and cytopathologist (MS). Regarding manually assessed samples with small cell numbers, 280 samples were observed using a light microscope (magnification, $x 40)$. In addition, the approximate number of cells on the whole slide was evaluated, and the samples were classified to contain $<1,000$ cells or $\geq 1,000$ cells. Thus, samples with $<$ one cell in one high-power field (HPF) were regarded as $<1,000$ cell cases because one HPF has an area of $0.785 \mathrm{~mm}^{2}$ and the whole area of the specimen $(2 \times 5 \mathrm{~cm})$ accounted for 1,273 HPFs. Moreover, the total number of cells in the whole slide of the specimen was estimated to be $<1,000$ cells if the number of cells per HPF was <one. The following samples were excluded from the CAIA procedure in the consensus meeting because the nuclei of leukocytes could not be appropriately detected: Samples with i) markedly denatured cells, ii) possible peripheral blood contamination, iii) $>50 \%$ red blood cells (RBCs), iv) a small number of cells $(<1,000)$, v) almost completely faded staining and vi) leukocyte aggregation (Fig. 1). Finally, CAIA was performed using 49 samples. Patients had a median age, 59.0 years; mean age, 55.8 years and age range, 20-80 years. Of the 49 patients 27 were male and 22 female. The clinical diagnosis/cytological diagnosis of the relevant patients are summarized in Table I as the comparison of original 280 cases and selected 49 cases.

Definition of groups used in the present study. The cases were grouped according to the combination of clinical and cytological diagnoses as follows: Cases with no tumor history or brain tumor according to CT, MRI or other modalities and with a confirmed negative diagnosis of CSF cytology (negative cytology) $\left(\mathrm{Clin}^{\text {non-tumor }} / \mathrm{Cyto}^{\text {negative }}\right)$, cases clinically suggestive of leptomeningeal carcinomatosis with negative cytology (Clin $\left.^{\text {tumor }} / \mathrm{Cyto}^{\text {negative }}\right)$ and cases clinically suggestive of leptomeningeal carcinomatosis with positive cytology (Clin ${ }^{\text {tumor/ }}$ Cyto $\left.^{\text {malignant }}\right)$. The present study included cytological cases in which the primary tumor type was confirmed by pathological diagnosis alone. The primary tumor histological type was classified according to the World Health Organization classification of tumors of the digestive system (20), the central nervous system (21), the lung, pleura, thymus and heart (22) and hematopoietic and lymphoid tissues (23) and is
Table I. Details of the clinical diagnosis/cytological diagnosis of cases.

\begin{tabular}{lcr}
\hline Clinical/cytological diagnosis & Before exclusion & CAIA \\
\hline All cases & 280 & 49 \\
Clin $^{\text {non-tumor} / C y t o ~}{ }^{\text {negative }}, \mathrm{n}(\%)$ & $120(43)$ & $10(21)$ \\
Non-HT, n $(\%)$ Clin ${ }^{\text {tumor }} / \mathrm{Cyto}^{\text {negative }}$ & $57(20)$ & $7(14)$ \\
Clin $^{\text {tumor }} / \mathrm{Cyto}^{\text {malignant }}$ & $17(6)$ & $12(24)$ \\
$\mathrm{HT}, \mathrm{n}(\%)$ & $65(23)$ & $7(14)$ \\
Clin $^{\text {tumor }} / \mathrm{Cyto}^{\text {negative }}$ & $21(8)$ & $13(27)$ \\
Clin $^{\text {tumor }} / \mathrm{Cyto}^{\text {malignant }}$ & & \\
\hline
\end{tabular}

CAIA, computer-assisted image analysis; Non-HT, non-hematological tumor; HT, hematological tumor; Clin, clinical; Cyto, cytology.

\begin{tabular}{|c|}
\hline Total $n=280$ cases of CSF cytology \\
\hline $\begin{array}{l}\text { Reason for exclusion } \\
\text { by microscopical analysis }\end{array}$ \\
\hline $\begin{array}{l}\text { - Markedly denatured cells }(n=3) \\
\text { - Possibility of peripheral } \\
\text { blood contamination }(n=66) \\
\text { - A small number of cells }(n=158) \\
\text { - Strong fading of staining }(n=2) \\
\text { - Aggregation of Leukocytes }(n=2\end{array}$ \\
\hline CAIA analyzed cases $(n=49)$ \\
\hline
\end{tabular}

Figure 1. Flowchart of computer-assisted image analysis cases. CSF, cerebrospinal fluid.

presented in Table II. In terms of tumor types, hematological malignancies were defined as described in the World Health Organization classification of tumors of hematopoietic and lymphoid tissues (23) as hematological tumor (HT) group, and all another tumors as non-hematological tumor (non-HT) group in the present study. The histological details of tumor types in CAIA analyzed tumors were analyzed and it was found that 5 out of 9 cases of non-HT group tumor belonged to primary CNS tumor, the other 4 cases belonged to metastatic carcinoma, 5 out of 7 HT group tumor belonged to malignant lymphoma, and other 2 cases belonged to leukemia (Table II).

WSI. The whole area of Pap-stained specimens and Giemsastained specimens of the same sample was captured using a virtual slide scanner (Nanozoomer SQ, Hamamatsu Photonics K.K.), and WSI was performed in the $\mathrm{x} 40$ mode (combination of 20X objective lens and $\mathrm{x} 2$ digital magnification). The specifications of the Nanozoomer SQ are as follows: Camera pixels, 12 million pixels; pixel size, $0.23 \mu \mathrm{m} /$ pixel; and LED source, tiling capture via a color CMOS sensor. Pap staining was focused manually, and Giemsa staining was focused automatically. 
Table II. Histological diagnosis of malignant cases in the present study.

\begin{tabular}{lc} 
Histological diagnosis & Cases, $\mathrm{n}(\%)$ \\
\hline Non-HT & \\
Brain medulloblastoma & $3(33)$ \\
Stomach poorly differentiated adenocarcinoma & $2(22)$ \\
Brain dysgerminoma & $1(11)$ \\
Brain malignant melanoma & $1(11)$ \\
Neuroendocrine carcinoma & $1(11)$ \\
(small-cell type) (rectum) & \\
Poorly differentiated NSCLC & $1(11)$ \\
HT & \\
All cases & \\
Malignant lymphoma & $5(71)$ \\
ALL & $1(14)$ \\
CML & $1(14)$
\end{tabular}

Non-HT, non-hematological tumor; NSCLC, non-small-cell lung cancer; HT, hematological tumor; ALL, acute lymphocytic leukemia; CML, chronic myelogenous leukemia.

CAIA. The WSI images of Pap- and Giemsa-stained specimens were acquired using a Panoramic Viewer version 1.15.4, QuantCenter, Histoquant module (3DHISTECK Ltd.). In addition, the protocols that could detect the nuclei of lymphocytes, neutrophils, macrophages, RBCs and all cells were determined independently, and each population was separately analyzed to enumerate the cell counts (Table III). The CAIA conditions for each blood cell type and the total cells are presented in Table III.

Accordance rate between CAIA and manual analysis. In total 29 representative cases were evaluated to assess the accuracy of CAIA. At least 50 leukocytes/case were evaluated. For this purpose, at least five images were captured (magnification, $\mathrm{x} 40$ ). If five images contained $<50$ leukocytes, additional images were captured until the total cell count reached at least 50 leukocytes. Then, one cytopathologist (M.S.) and one cytotechnologist (S.K.) manually evaluated the images, and consensus meetings were held to determine the cell types. Then, the same images were utilized for CAIA. A two-way evaluation was performed involving the accuracy of CAIA in evaluating manually evaluated leukocytes (power of analysis) and the accuracy of CAIA-detected cells under each condition shown in Table III in comparison with manually evaluated cells (accuracy of analysis condition).

Statistical analysis. JMP Pro version 12.2.0 software (SAS Institute Inc.; https://www.jmp.com/en_us/home.html) was used for all statistical analyses. The Wilcoxon rank-sum test was used for comparisons between the total cells of Pap- and Giemsa-stained specimens. P-value was calculated by one-way chi-square approximation for Wilcoxson ranksum test. For multiple comparisons, Wilcoxon's test should not be repeated, thus the Steel-Dwass test was used for multiple comparisons involving each blood cell component for each clinical diagnosis/cytological diagnosis in the nonhematological tumor (non-HT) and hematological tumor (HT) groups (24). Asymptotic test was performed to calculate the P-values in Steel-Dwass test. For the statistical examination of contingency tables in which one of the groups was $<5$, the Fisher's exact test was used. $\mathrm{P}<0.05$ was considered to indicate a statistically significant difference. As the present sample size was relatively small, in addition to the aforementioned significant level, the power of the statistical analysis was presented as 1- $\beta$ using JMP Pro version 12.2.0 software. The cut-off for the population of macrophages in the cytological diagnosis was calculated using the Youden index (25) and the area under the curve (AUC) was estimated.

\section{Results}

Increased total cell count in malignant cytological CSF specimens using manual evaluation. All Giemsa-stained specimens were initially analyzed by CAIA. However, due to several reasons summarized in Fig. 1, 231 cases could not be analyzed by CAIA. The main reasons were a small number of cells $(n=158)$ and possibility of peripheral blood contamination $(n=66)$, which account for $97.0 \%$ of excluded cases (Fig. 1). Comparison of 280 cases and CAIA analyzed 49 cases for clinical diagnosis and cytological diagnosisbased grouping were shown in Table I. The association between the cell count in Giemsa specimens and the clinical background, cytological diagnosis and tumor type (HT or non-HT) was established via manual estimation (Fig. 2). In the non-HT group, the percentages of samples with cell counts of $>1,000$ cells were $28.3 \%(34 / 120)$ in the Clin ${ }^{\text {non-tumor/ }}$ Cyto $^{\text {negative }}$ group, $50.9 \%(29 / 57)$ in the $\mathrm{Clin}^{\text {tumor }} / \mathrm{Cy}^{\text {to }}{ }^{\text {negative }}$ group and $100 \%(17 / 17)$ in the $\mathrm{Clin}^{\text {tumor }} / \mathrm{Cyto}^{\text {malignant }}$ group (Fig. 2A). In the HT group, the percentages of samples containing $>1,000$ cells were 28.3 (34/120), 36.9 (24/65), and $85.7 \%$ (18/21) in the $\mathrm{Clin}^{\text {non-tumor }} / \mathrm{Cyto}^{\text {negative }}, \mathrm{Clin}^{\text {tumor }} / \mathrm{Cyto}^{\text {negative }}$ and $\mathrm{Clin}^{\text {tumor }} / \mathrm{Cyto}^{\text {malignant }}$ groups, respectively (Fig. 2B). These data suggested frequency of cases that can be analyzed by CAIA were high in cytologically malignant cases in both the HT and non-HT groups.

Giemsa staining is suitable for whole slide leukocyte counting via CAIA. To compare the total cell number between WSI and CAIA for Giemsa and Pap specimens prepared from the same samples, the whole images of Pap and Giemsa specimens were analyzed with respect to the number of nuclei to detect individual cells (Fig. 3). The average total cell counts in Pap and

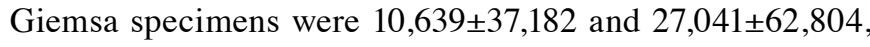
respectively; thus, the cell count was 2.54-fold higher in Giemsa specimens $(\mathrm{P}<0.0001)$. These data suggested that Giemsa specimens were superior to Pap smear in terms of cell holding ability on the glass.

An increased percentage of macrophages in cytological CSF specimens is an indicator of non-HT tumors. For the 49 samples subjected to CAIA, the number and subtype ratio of the leukocyte population was evaluated. The percentage of each leukocyte subtype was assessed according to the combination of the clinical diagnosis at the time of sample submission and the cytological diagnosis of the specimens using the 
Table III. Image analysis conditions for each blood cell type and all cells.

\begin{tabular}{lccccc}
\hline & \multicolumn{3}{c}{ Cell type } \\
\cline { 2 - 4 } Factor & Lymphocytes & Lymphocytes & Macrophages & RBCs & All cells \\
\hline Cell size, pixels & $20-70$ & $35-70$ & $71-140$ & $15-30$ & $20-1,000$ \\
Shape factor & $0.51-1$ & $0-0.5$ & $0-1$ & $0.7-1$ & $0-1$ \\
Red range & $57-224$ & $57-224$ & $48-241$ & $60-240$ & $48-241$ \\
Green range & $25-172$ & $25-172$ & $45-156$ & $113-208$ & $25-172$ \\
Blue range & $85-205$ & $85-214$ & $111-222$ & $120-215$ & $85-222$ \\
\hline
\end{tabular}

RBC, red blood cell.

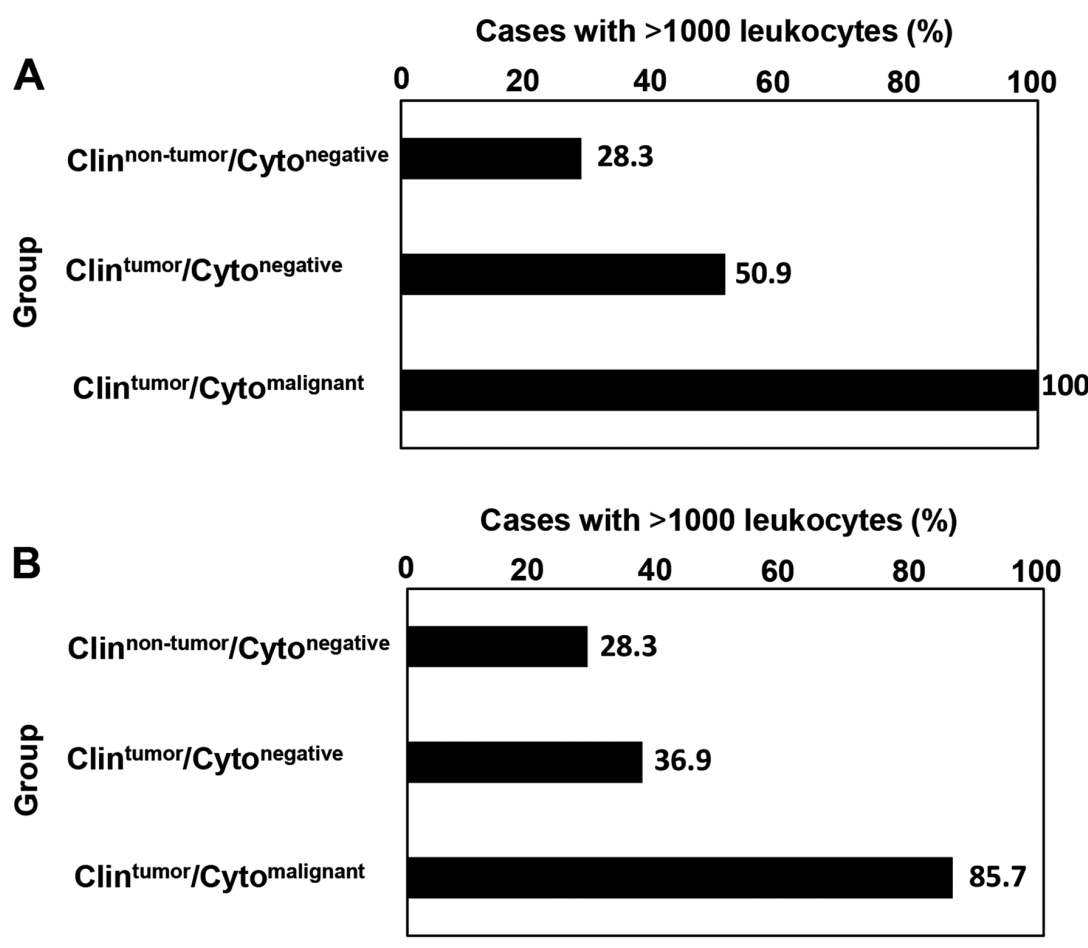

Figure 2. Frequency of cases with $>1,000$ leukocytes evaluated via manual observation based on the combination of the clinical background and cytological diagnosis. (A) Non-hematological tumor cases were analyzed as tumor cases. (B) Hematological tumor cases were analyzed as tumor cases. Clin, clinical; Cyto, cytology.

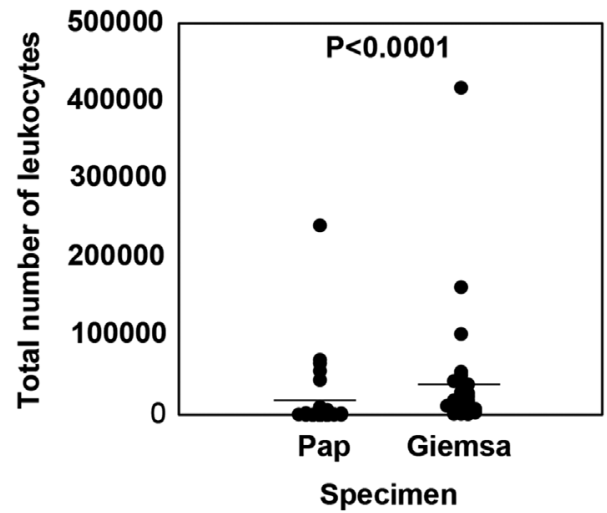

Figure 3. Comparison of the total number of leukocytes between Pap- and Giemsa-stained specimens using the Wilcoxon rank-sum test $(n=49)$. Leukocyte nuclei were assessed via computer-assisted image analysis, and the whole area of the specimens was examined to determine the number of detected nuclei for Giemsa and Pap specimens. Pap, Papanicolaou.
Steel-Dwass test. Next, samples from cases of malignancy in the non-HT (Fig. 4A-F) were evaluated and multiple comparisons among the clinical diagnosis/cytological diagnosis groups were conducted. In the non-HT group, the lymphocyte ratio was significantly different between the Clin ${ }^{\text {non-tumor/ }}$ $\mathrm{Cyto}^{\text {negative }}$ and $\mathrm{Clin}{ }^{\text {tumor }} / \mathrm{Cyto}^{\text {malignant }}$ groups $(\mathrm{P}=0.0061, \alpha=5 \%$, $1-\beta=57.30 \%)$ but not between the $\mathrm{Clin}^{\text {non-tumor }} / \mathrm{Cyto}^{\text {negative }}$ and $\mathrm{Clin}^{\text {tumor }} / \mathrm{Cy}_{\text {to }}{ }^{\text {negative }}$ groups $(\mathrm{P}=0.2022)$ or between the $\mathrm{Clin}^{\text {tumor/ }}$ Cyto $^{\text {negative }}$ and $\mathrm{Clin}^{\text {tumor }} /$ Cyto $^{\text {malignant }}$ groups $(\mathrm{P}=0.4888$; Fig. 4A). The neutrophil ratio was not significantly different among the three groups (Fig. 4C). Meanwhile, the macrophage ratio significantly differed between the $\mathrm{Clin}^{\text {non-tumor }} / \mathrm{Cyto}^{\text {negative }}$ and $\mathrm{Clin}^{\text {tumor }} / \mathrm{Cyto}^{\text {negative }}$ groups $(\mathrm{P}=0.0343, \alpha=5 \%, 1-\beta=61.94 \%)$ and between the $\mathrm{Clin}^{\text {non-tumor }} / \mathrm{Cy}$ to ${ }^{\text {negative }}$ and $\mathrm{Clin}{ }^{\text {tumor }} / \mathrm{Cyto}^{\text {malignant }}$ groups $(P=0.0299, \alpha=5 \%, 1-\beta=24.45 \%)$ but not between the $\mathrm{Clin}^{\text {tumor }} / \mathrm{Cy}$ to $^{\text {negative }}$ and $\mathrm{Clin}{ }^{\text {tumor }} / \mathrm{Cy}$ to ${ }^{\text {malignant }}$ groups $(\mathrm{P}=0.4381$; Fig. 4E). Representative images obtained using 


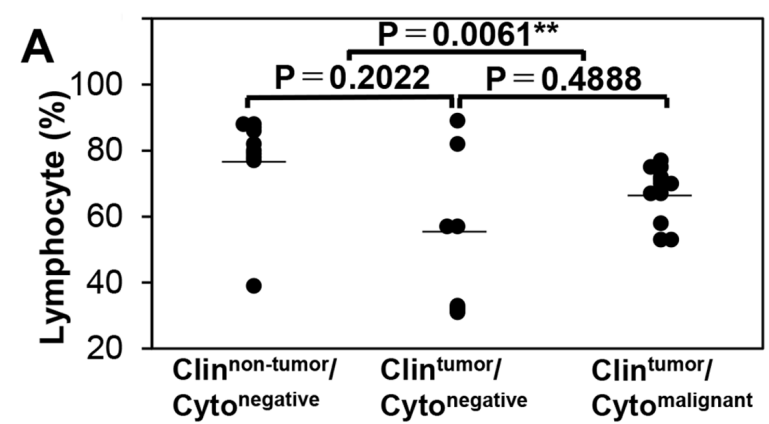

B

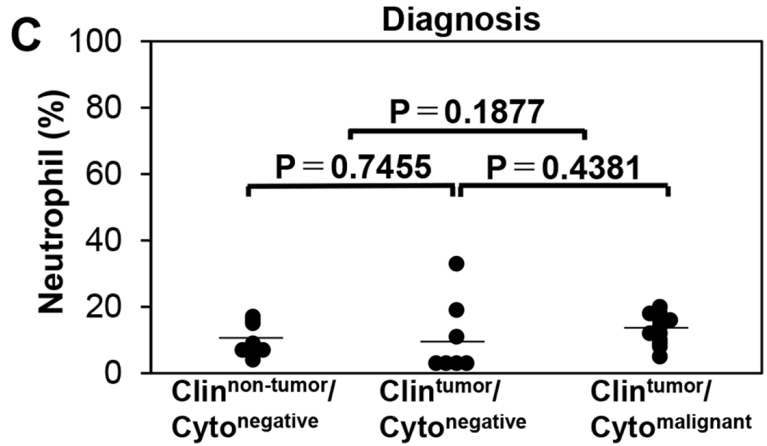

D

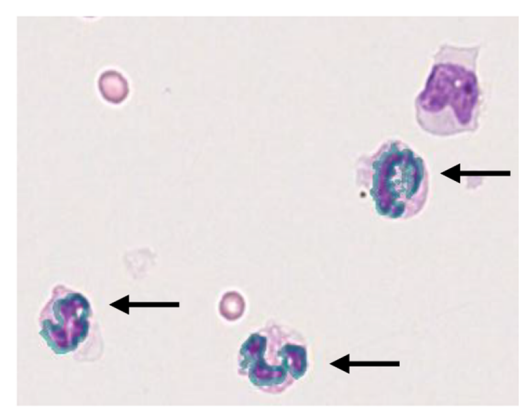

E

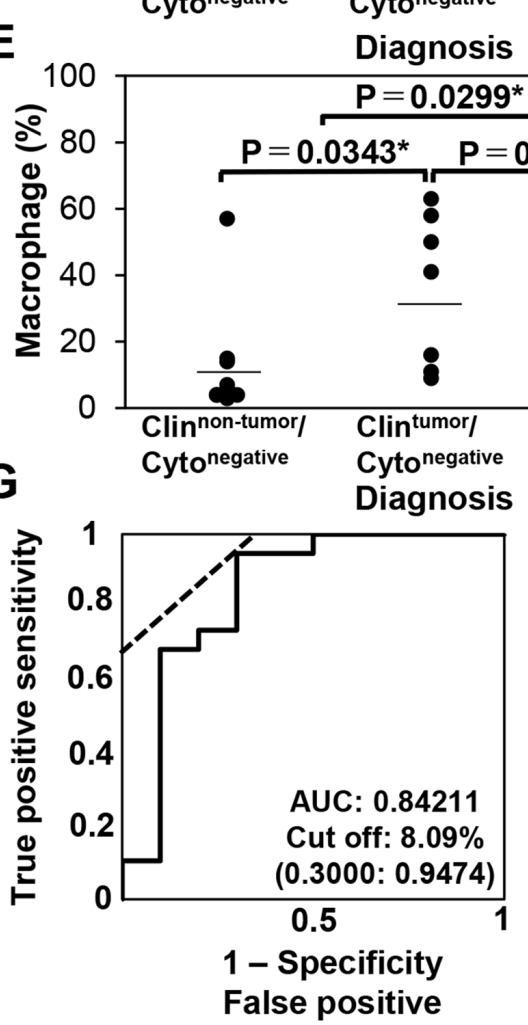

$\mathbf{F}$

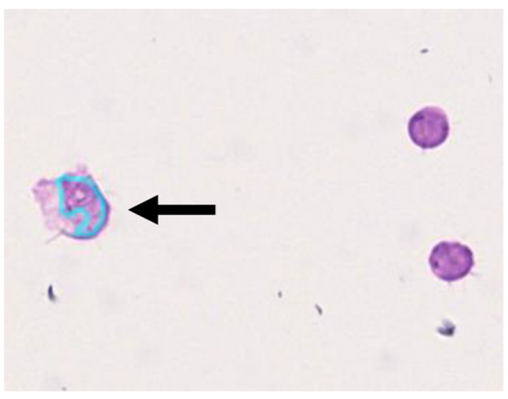

Cytomalignant

H

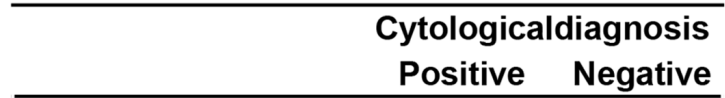

\begin{tabular}{|c|c|c|c|}
\hline \multirow{2}{*}{$\begin{array}{l}\text { Macrophage } \\
(\%)\end{array}$} & $\geq 8.09 \%$ & 11 & 10 \\
\hline & $<8.09 \%$ & 1 & 7 \\
\hline \multicolumn{2}{|c|}{ Total } & 12 & 17 \\
\hline \multirow{2}{*}{\multicolumn{3}{|c|}{$\begin{array}{l}\text { Sensitivity } \\
\text { Specificitv }\end{array}$}} & $92 \%$ \\
\hline \multirow{2}{*}{\multicolumn{3}{|c|}{$\begin{array}{l}\text { Specificity } \\
\text { Likelihood ratio }\end{array}$}} & $41 \%$ \\
\hline & & & 1.56 \\
\hline \multicolumn{3}{|c|}{ The two-sided $P$ value $\#$} & 0.0926 \\
\hline
\end{tabular}

Figure 4. Multiple comparisons of the leukocyte ratio (\%) using the Steel-Dwass test in Giemsa-stained cerebrospinal fluid specimens for the Clin ${ }^{\text {non-tumor/ }}$ Cyto $^{\text {negative }}(\mathrm{n}=10), \mathrm{Clin}^{\text {tumor }} / \mathrm{Cyto}^{\text {negative }}(\mathrm{n}=7)$ and $\mathrm{Clin}^{\text {tumor }} / \mathrm{Cyto}^{\text {malignant }}(\mathrm{n}=12)$ groups of non-hematological tumor samples. (A and B) Percentage for lymphocytes and representative image of lymphocytes detected by CAIA. (C and D) Percentage of neutrophils and representative image of neutrophils detected by CAIA. (E and F) Percentage of macrophages and representative image of macrophages detected by CAIA. (Giemsa staining, x 20 magnification). The detected lymphocytes are circled by a red line, neutrophils are circled by a green line (thin arrow), and detected macrophages are circled by a blue line (thick arrow). (G) The receiver operating characteristic curve of the macrophage ratio (\%) for distinguishing the Clinn ${ }^{\text {non-tumor }} /$ Cyto ${ }^{\text {negative }}$ group from the other groups (H) Accuracy of the Cut-off of the macrophage ratio (\%) in comparison to the cytological diagnosis. ${ }^{*} \mathrm{P}<0.05,{ }^{* *} \mathrm{P}<0.01$, significant according to the Steel-Dwass test. ${ }^{~} \mathrm{P}<0.05$, Fisher's exact test. Clin, clinical; Cyto, cytology; AUC area under the curve.

CAIA are presented in Fig. 4B, D and F. As the macrophage population was higher in the $\mathrm{Clin}^{\text {non-tumor }}$ group compared with $\mathrm{Clin}^{\text {tumor }}$ group including $\mathrm{Clin}^{\text {tumor}} / \mathrm{Cy}^{\text {to }}{ }^{\text {negative }}$ and $\mathrm{Clin}^{\text {tumor}} /$ Cyto ${ }^{\text {malignant }}$ group, the association between the ratio of macrophages among total leukocytes and clinical negativity was analyzed. The receiver operating characteristic curve of the macrophage ratio for distinguishing the $\mathrm{Clin}^{\text {non-tumor }} / \mathrm{Cyto}^{\text {negative }}$ group from the clinically non-HT $\approx$ group (including both cytologically negative and positive cases) was examined, and the cut-off was calculated. As presented in Fig. 4G, the AUC 
Table IV. Average accordance rate between CAIA and manual evaluation for each leukocyte type for 29 samples.

A, Accordance rate of CAIA with manual evaluation

Lymphocytes, \% (Mean/SD) Neutrophils, \% (Mean/SD) $\quad$ Macrophages, \% (Mean/SD) All leukocytes, \% (Mean/SD)

$\begin{array}{llll}70.53(39.3 / 17.1) & 6.37(3.7 / 15.4) & 59.62(14.9 / 11.4) & 68.33(57.9 / 34.5)\end{array}$

$\mathrm{B}$, Accordance rate of manual with CAIA evaluation

Lymphocytes, \% (Mean/SD) Neutrophils, \% (Mean /SD) $\quad$ Macrophages, \% (Mean /SD) All leukocytes, \% (Mean/SD)

\begin{tabular}{llll}
\hline $79.14(35.2 / 19.9)$ & $7.52(3.9 / 6.8)$ & $65.26(14.3 / 19.0)$ & $67.30(53.3 / 33.2)$
\end{tabular}

CAIA, computer-assisted image analysis.

was 0.84211 , and the cut-off was $8.09 \%$. Moreover, the likelihood ratio was 1.56 , and the P-value using the Fisher's exact test was 0.0926 (Fig. 4H). These results suggested that the macrophage population was increased in the non-HT tumor group and that this increase could be detected by CAIA.

Malignant hematological tumors and the reactive leukocyte population in cytological CSF specimens cannot be distinguished via CAIA. Next, the leukocyte population in the HT group was evaluated, and it was reported that the lymphocyte ratio did not differ among any of the groups (Fig. 5A). Meanwhile, the neutrophil ratio significantly differed only between the $\mathrm{Clin}^{\text {tumor }} / \mathrm{Cy}$ to $^{\text {negative }}$ and $\mathrm{Clin}^{\text {tumor}} / \mathrm{Cy}$ to ${ }^{\text {malignant }}$ groups $(\mathrm{P}=0.0153$; Fig. 5B). In addition, the macrophage ratio did not differ among the groups (Fig. 5C). The representative malignant lymphoma sample in which tumor nuclei were incorrectly identified as normal lymphocytes or macrophages is presented in Fig. 6A-C. In this case, under both the lymphocyte, and macrophage detection conditions, more than half of the lymphoma cells were incorrectly recognized as normal. These data indicated that in the present condition of CAIA, leukocytes should not be evaluated in HT tumor cases.

Macrophage population is increased in non-HT cases using manual and CAIA evaluation. To evaluate the power and accuracy of specific subtype analyses, the rate of accordance between CAIA and manual analysis in the non-HT group was compared (Table IV). The average accordance rate of CAIA with manual evaluation was $68.33 \%$ for total leukocyte counts in the non-HT cases analyzed in Fig. $4(n=29)$. Conversely, the average accordance rate of manual evaluation with CAIA was $67.30 \%$ for total leukocyte count in the same cases. In addition, the average accordance rate of CAIA with manual evaluation and that of manual evaluation with CAIA were 70.53 and $79.14 \%$, respectively, for lymphocyte counts and 59.62 and $65.26 \%$, respectively, for macrophage counts (Table IV). However, the average accordance rate of CAIA with manual evaluation and that of manual evaluation with CAIA were very low (6.37 and $7.52 \%$, respectively) for neutrophil counts, primarily due to the low number of cells and misrecognition of neutrophils as macrophages (Table IV). As the accordance rate between CAIA and manual
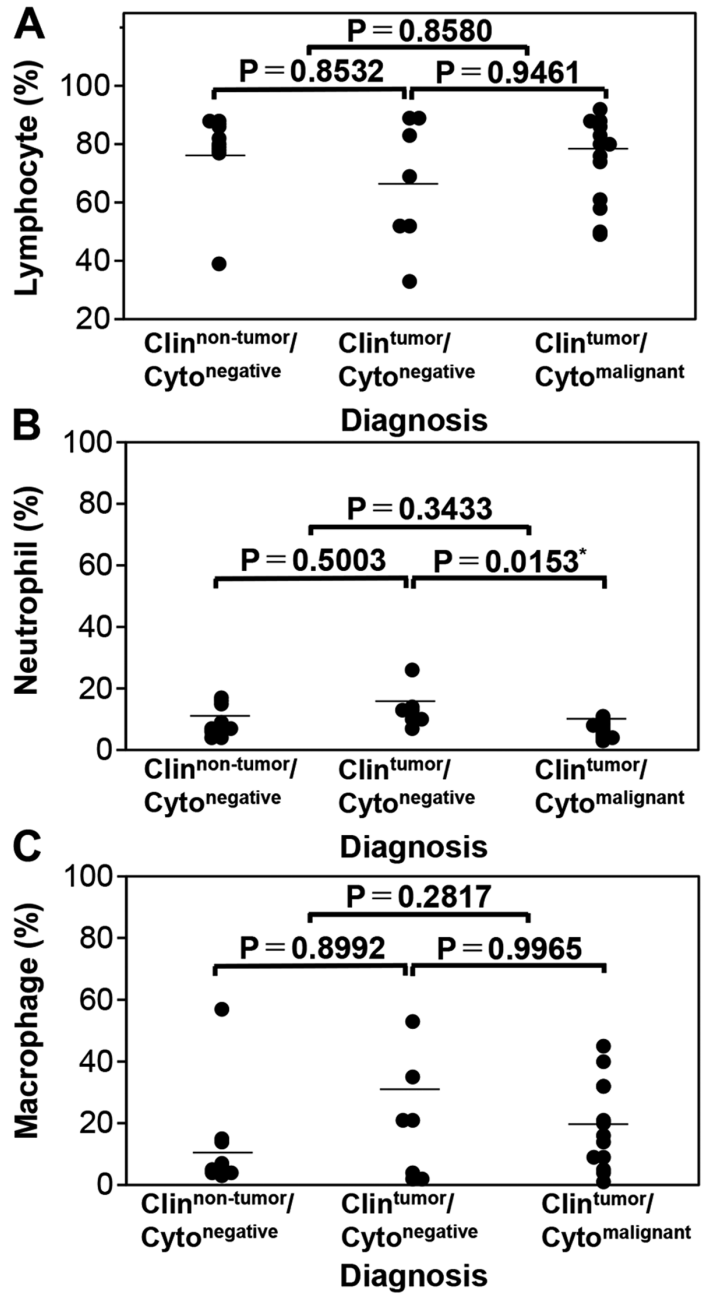

Figure 5. Multiple comparison of each leukocyte type (\%) using the SteelDwass test in Giemsa-stained cerebrospinal fluid specimens in the Clin ${ }^{\text {non-tumor }}$ Cyto $^{\text {negative }}(\mathrm{n}=10), \mathrm{Clin}^{\text {tumor }} / \mathrm{Cyto}^{\text {negative }}(\mathrm{n}=7)$ and $\mathrm{Clin}^{\text {tumor }} / \mathrm{Cyto}^{\text {malignant }}(\mathrm{n}=13)$ groups of hematological tumor samples. Percentage of (A) lymphocytes, (B) neutrophils and (C) macrophages. "Significant by the Steel-Dwass test. Clin, clinical; Cyto, cytology.

evaluation was not high, the specimens of non-HT cases (Fig. 7A-C) was also evaluated and multiple comparisons were performed among the clinical/cytological diagnosis groups. 


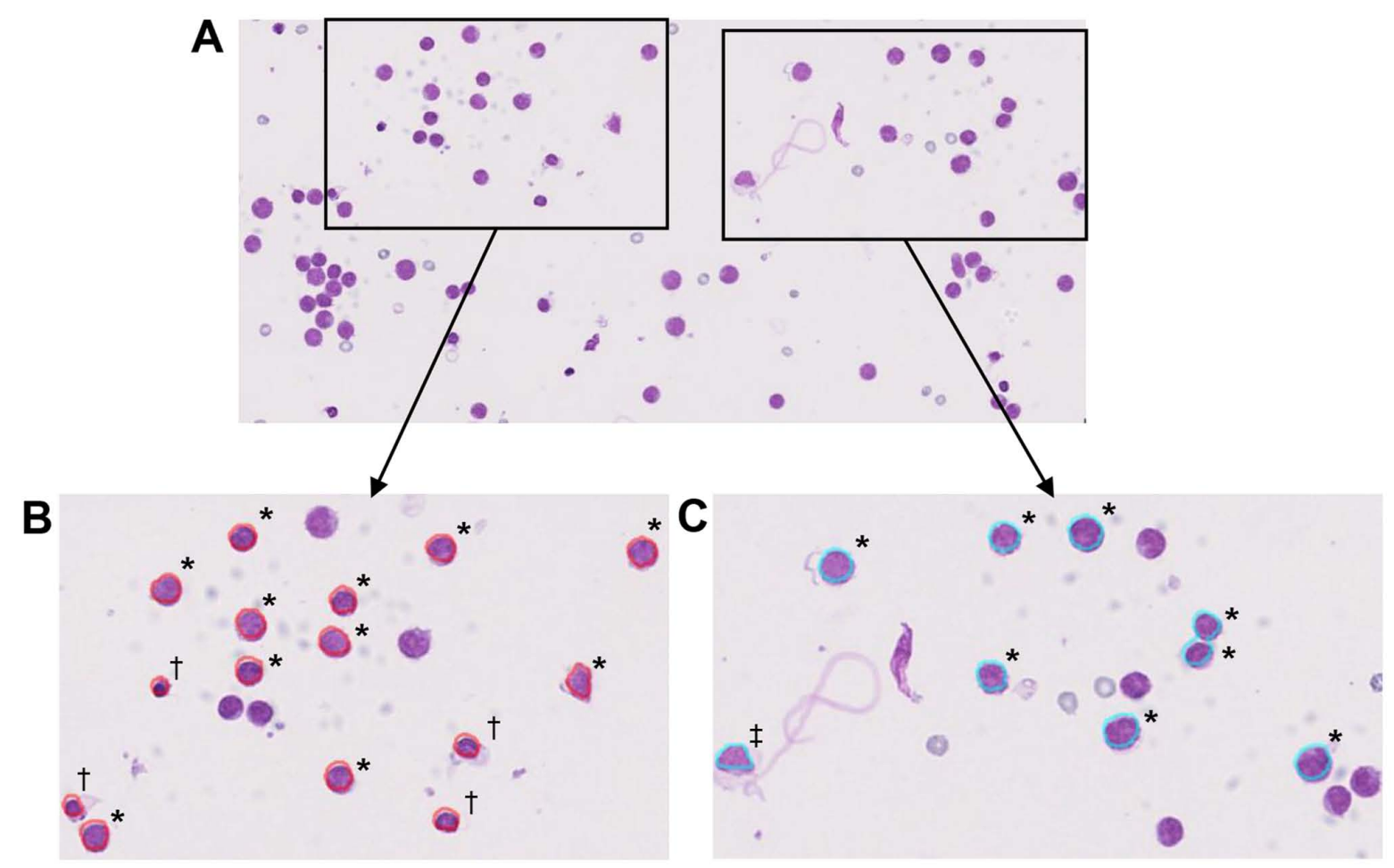

Figure 6. Representative images of hematological tumor samples. Clinical and cytological diagnosis of this case was malignant lymphoma. (A) Before (Giemsa staining, x20 magnification) and after computer-assisted image analysis under conditions for detecting (B) lymphocytes and (C) macrophages (Giemsa staining, x40 magnification). Incorrectly recognized lymphoma cells are circled by a red (B) lymphocyte detection setting) or blue line (C) macrophage detection setting). "Normal lymphocyte. ${ }^{\dagger}$ Normal macrophage. "Lymphoma cell.

In the non-HT group, the lymphocyte ratio was significantly different between the $\mathrm{Clin}^{\text {non-tumor}} / \mathrm{Cyto}^{\text {negative }}$ and $\mathrm{Clin}^{\text {tumor}} /$ Cyto $^{\text {malignant }}$ groups $(\mathrm{P}=0.0249 ; \alpha=5 \% ; 1-\beta=69.08 \%)$ but not between the $\mathrm{Clin}^{\text {non-tumor }} / \mathrm{Cy}^{\text {to }}{ }^{\text {negative }}$ and $\mathrm{Clin}^{\text {tumor }} / \mathrm{Cy}^{\text {to }}{ }^{\text {negative }}$ groups $(\mathrm{P}=0.2207)$ or between the $\mathrm{Clin}^{\text {tumor }} / \mathrm{Cyto}^{\text {negative }}$ and Clin ${ }^{\text {tumor }} /$ Cyto $^{\text {malignant }}$ groups $(\mathrm{P}=0.6483)$ (Fig. 7A). The neutrophil ratio did not significantly differ among the groups (Fig. 7B). Meanwhile, the macrophage ratio significantly differed between the $\mathrm{Clin}^{\text {non-tumor}} / \mathrm{Cyto}^{\text {negative }}$ and $\mathrm{Clin}^{\text {tumor }} /$ Cyto $^{\text {malignant }}$ groups $(\mathrm{P}=0.0061 ; \alpha=5 \% ; 1-\beta=96.82 \%)$, but not between the $\mathrm{Clin}^{\text {non-tumor}} / \mathrm{Cy}^{\text {to }}{ }^{\text {negative }}$ and $\mathrm{Clin}^{\text {tumor }} / \mathrm{Cyto}^{\text {negative }}$ groups $(\mathrm{P}=0.1378)$ or between the $\mathrm{Clin}^{\text {tumor}} / \mathrm{Cyto}^{\text {negative }}$ and $\mathrm{Clin}^{\text {tumor }} / \mathrm{Cyto}^{\text {malignant }}$ groups $(\mathrm{P}=0.6483$; Fig. 7C). These data suggested that, although the accordance rate between CAIA analysis and manual evaluation was not high, there was a tendency of a decreased lymphocyte population and an increased macrophage population in both evaluation methods. Overall, these data suggested that increased percentage of monocyte/macrophage population within the total leukocyte population in Giemsa-stained specimens should be considered as a sign of non-HT leptomeningeal carcinomatosis, at least in medulloblastoma, poorly differentiated adenocarcinoma of the stomach, dysgerminoma of the brain, malignant melanoma of the brain, neuroendocrine carcinoma (small-cell type) of the rectum and poorly differentiated non-small cell lung carcinoma which was the cases of non-HT tumors as summarized in Table II. Therefore, our data indicated that an increase in the monocyte/macrophage population in cytological specimens should prompt evaluation for the possible presence of leptomeningeal carcinomatosis in patients with these tumors.

\section{Discussion}

The present study emphasized the significance of the examination of the background leukocyte population in cytological specimens using CAIA. First, the association between the number of cells in cytological CSF specimens and the cytological diagnosis (benign or malignant) was examined. The frequency of specimens containing $>1,000$ cells was increased in malignant cases regardless of the tumor type. Regarding the cell count in CSF, several reports have indicated that an increased cell count is observed in the malignant CSF group via manual $(4,6,7)$ and flow cytometric evaluation (9). In the present study, the number of cells on the glass slide did not reflect the number of cells per volume of CSF because the amount of CSF used varied between cases; however, the number of cells might have been increased in cytologically positive cases. The CSF volume used to prepare the specimens could not be determined from medical records. Thus, when recording the amount of CSF submitted to the laboratory, the amount of CSF used to prepare specimens should be noted, and the leukocyte composition of cytological specimens is important.

Next, it was determined whether Giemsa or Pap staining was the optimal preparative method for CAIA. The number of cells was significantly higher for Giemsa samples compared with those of Pap samples. Anand et al (26) reported that hematoxylin and eosin-stained and Pap-stained specimens had fewer cells loaded on the glass, whereas this was not observed for Giemsa-stained specimens because the cells adhered more firmly to the glass slide during dry fixation. Beyer-Boon et al (27) reported that more cells remain on the 

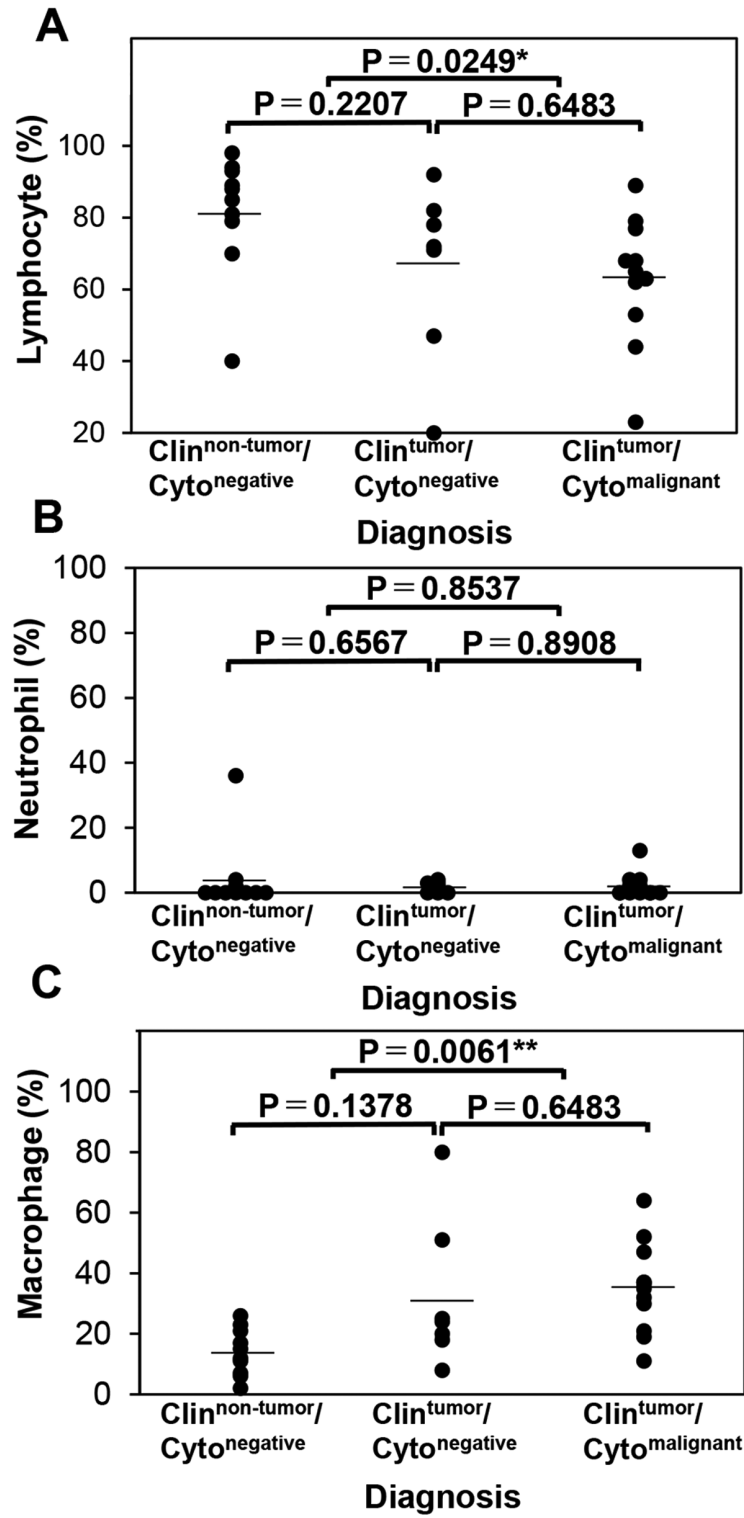

Figure 7. Multiple comparison of each leukocyte type (\%) by manual evaluation using the Steel-Dwass test in Giemsa-stained cerebrospinal fluid specimens in the $\mathrm{Clin}^{\text {non-tumor}} / \mathrm{Cyto}^{\text {negative }}(\mathrm{n}=10), \mathrm{Clin}^{\text {tumor }} / \mathrm{Cy} \mathrm{to}^{\text {negative }}(\mathrm{n}=7)$ and $\mathrm{Clin}^{\text {tumor }}$ Cyto $^{\text {malignant }}(n=12)$ groups of non-hematological tumor samples. Percentage of (A) lymphocytes, (B) neutrophils and (C) macrophages. ${ }^{*} \mathrm{P}<0.05,{ }^{* *} \mathrm{P}<0.01$, significant according to the Steel-Dwass test. Clin, clinical; Cyto, cytology.

glass in Giemsa (dry fixation) specimens compared with those in Pap (alcohol fixation) specimens among urine cytology cases. These results are in agreement with the present findings. Thus, Giemsa specimens are suitable for the leukocyte evaluation of cytological CSF specimens using CAIA.

Then, the association between the percentage of each leukocyte type and the cytological diagnosis was determined. Among samples from the non-HT group, the lymphocyte ratio was significantly decreased, macrophage ratio was significantly increased and there was no difference in the neutrophil ratio between the cytologically positive and cytologically negative groups. In addition, the possibility of distinguishing between the cytologically tumor-negative and tumor-positive cases based on the cut-off macrophage ratio was demonstrated. Similar studies focusing on inflammatory cells have been reported by Djukic et al (7) and Illan et al (9).
Djukic et al described the relation of total cell density (include tumor cells and leukocytes) and frequency of lymphocytes and granulocytes in the CFS, and lymphocytes and granulocytes were frequently observed in total cell number high cases ( $>4$ cells/ $\mu$ l cases). However, they just examined the cases of metastatic carcinomas, hematological malignancies and primary CNS tumors altogether. Djukic et al used a different grouping of cases compared with the current study, and did not mention percentages of leukocyte subtypes, thereby making it difficult to compare the results. Illan et al compared the percentages of lymphocytes, neutrophils and monocytes between cytologically negative and positive groups; however, the percentage of neutrophils was significantly increased, whereas those of lymphocytes and monocytes were not significantly different. These results are not identical to those in the present study. A possible explanation for this may be that Illan et al used clinically malignant/cytologically negative samples, unlike the negative group of the present study. In the present study, the macrophage ratio was high in the non-HT cytologically positive group compared with cytologically negative group. In the tumor microenvironment, tumor-associated macrophages (TAMs) infiltrate and promote tumor progression (28). Monocytes in the peripheral blood are recruited to the tumor site and differentiate into macrophages in response to chemokines and growth factors produced by tumor cells (28). Among the non-HT samples examined in the present study, the type of factors that contributed to the TAM recruitment for gastric cancer, medulloblastoma and dysgerminoma could not be identified. Reportedly, TAMs are recruited by chemokines produced by non-small cell lung cancer cells, homeoproteins produced by colon cancer cells, and secreted and transmembrane protein 1 produced by melanoma cells (29-31). These reports suggest that macrophage recruitment is increased by tumor-produced factors. Thus, the increased percentage of macrophages in cytological CSF specimens in clinically malignant cases reflects TAM induction. To the best of our knowledge, no studies have examined the association between cytokine/chemokine production in the tumor microenvironment in the central nervous system and the leukocyte population in the CSF. Thus, this association should be examined in the future.

In terms of normal CSF, the median leukocyte ratios are $86.5 \%$ for lymphocytes, $10.5 \%$ for monocytes and $2.0 \%$ for macrophages (3). In leptomeningeal carcinomatosis, the median leukocyte ratios are $59.7 \%$ for lymphocytes, $24.0 \%$ for monocytes and $1.5 \%$ for neutrophils (9). Thus, these reports support the present findings demonstrating that the macrophage population is increased in non-HT tumor group. The present data suggested that an increased number of macrophages is an indicator of leptomeningeal carcinomatosis in cytological specimens of leptomeningeal spaces even in atypical cells are not identified. Indeed, Chamberlain et al (32) reported that the cytological detection of malignant cells in leptomeningeal metastasis is affected by the collection site, for example ventricular or lumbar CSF. Thus, in cases where intracranial tumors are detected, it is possible that spinal samples will be negative, and the converse, that is, spinal tumor cases with intracranial sample will be negative can also be true (32). Thus, cytopathologists and cytotechnologists should monitor patients for leptomeningeal carcinomatosis when they detect 
a higher macrophage count in CSF samples. In order to investigate the possibility that macrophage infiltration into CSF can occur in advance of malignant cells exuding into the CSF, chronologically collected CSF samples of the same patients are used. However, the present study could not evaluate the cytological specimens of the same patients chronologically due to lack of cases. Future studies including chronological analysis are necessary to confirm monocyte/macrophage characteristics and to assess changes in their numbers with the emergence of tumor cells in the CSF.

Finally, the adequacy of the sample size for statistical analysis must be discussed. In statistical analysis, two types of errors exist (33). One is $\alpha$ errors (type I errors), in which a significant difference is incorrectly identified between two groups (33). The other is $\beta$ errors (type II errors), in which the absence of a significant difference is incorrectly identified between two groups (33). Indeed, $\alpha$ for the present statistically significant results was $<5 \%$. Thus, there was a low possibility of a type I error. Usually, 1- $\beta$ represents the power of a statistical test. In statistical tests, the power increases as the sample size increases (34). Unfortunately, the present results exhibited low power, indicating the possibility that the data were incorrectly considered non-significant. Therefore, larger sample sizes should be used in future research.

Overall, the analysis of the cytological specimens in the present study revealed that leukocyte counts in the background were higher in cytologically positive cases, and the percentage of macrophages was elevated in non-HT cases. Therefore, in the future, it is important to focus on the number of leukocytes and the leukocyte ratio in the background while examining cytological CSF specimens.

\section{Acknowledgements}

Not applicable.

\section{Funding}

This study was supported by the annual experimental budget to MS and SK given by Gunma University.

\section{Availability of data and materials}

The datasets used and/or analyzed during the current study are available from the corresponding author on reasonable request.

\section{Authors' contributions}

SK conducted clinical data collection, digital imaging of the specimens via virtual slide scanning, image analysis, statistical analysis, figure preparation and manuscript preparation. MS developed the experimental design, conducted experiments, and performed digital imaging of specimens via virtual slide scanning, image analysis, figure preparation, statistical analysis and manuscript preparation. MF assisted with clinical data collection and reviewed the manuscript for manuscript preparation. $\mathrm{JH}, \mathrm{TO}$ and $\mathrm{TF}$ diagnosed independently by the request of MS for the cases which original diagnosis and diagnosis in this study at consensus meeting by SK and MS was not identical. JH, TO and TF also reviewed the manuscript for manuscript preparation. All authors read and approved the manuscript and agreed to be accountable for all aspects of the research to ensure that the accuracy or integrity of any part of the work was appropriately investigated and resolved.

\section{Ethics approval, consent to participate}

The present study was approved by the Gunma University Ethical Review Board for Medical Research Involving Human Subjects of Gunma University School of Medicine (GUERB; Gunma, Japan) (approval number, HS2017-120), and the written notification for the current study was presented publicly on the webpage of Gunma University Hospital. Furthermore, the possibility to decline participation in this study was provided according to the Ethical Guidelines for Medical and Health Research Involving Human Subjects of the Japanese government (Ministry of Education, Culture, Sports, Science and Technology, and Ministry of Health, Labour and Welfare). Informed consent was waived by GUERB based on the above guidelines due to the retrospective nature of the study.

\section{Patient consent for publication}

Not applicable.

\section{Competing interests}

The authors declare that they have no competing interests.

\section{References}

1. Roth P and Weller M: Management of neoplastic meningitis. Chin Clin Oncol 4: 26, 2015.

2. Chamberlain MC: Neoplastic meningitis. Oncologist 13:967-977, 2008.

3. Sornas R: The cytology of the normal cerebrospinal fluid. Acta Neurol Scand 48: 313-320, 1972.

4. Rahimi J and Woehrer A: Overview of cerebrospinal fluid cytology. Handb Clin Neurol 145: 563-571, 2017.

5. Hayward RA, Shapiro MF and Oye RK: Laboratory testing on cerebrospinal fluid. A reappraisal. Lancet 1: 1-4, 1987.

6. MacKenzie JM: Malignant meningitis: A rational approach to cerebrospinal fluid cytology. J Clin Pathol 49: 497-499, 1996.

7. Djukic M, Trimmel R, Nagel I, Spreer A, Lange P, Stadelmann C and Nau R: Cerebrospinal fluid abnormalities in meningeosis neoplastica: A retrospective 12-year analysis. Fluids Barriers CNS 14: 7, 2017.

8. van Zanten AP, Twijnstra A and Ongerboer de Visser BW: Routine investigations of the CSF with special reference to meningeal malignancy and infectious meningitis. Acta Neurol Scand 77: 210-214, 1988.

9. Illan J, Simo M, Serrano C, Castañón S, Gonzalo R, MartínezGarcía M, Pardo J, Gómez L, Navarro M, Altozano JP, et al: Differences in cerebrospinal fluid inflammatory cell reaction of patients with leptomeningeal involvement by lymphoma and carcinoma. Transl Res 164: 460-467, 2014.

10. Singh G, Mathur SR, Iyer VK and Jain D: Cytopathology of neoplastic meningitis: A series of 66 cases from a tertiary care center. Cytojournal 10: 13, 2013.

11. Ho CY, VandenBussche CJ, Huppman AR, Chaudhry R and Ali SZ: Cytomorphologic and clinicoradiologic analysis of primary nonhematologic central nervous system tumors with positive cerebrospinal fluid. Cancer Cytopathol 123: 123-135, 2015.

12. Rao R, Hoda SA, Marcus A and Hoda RS: Metastatic breast carcinoma in cerebrospinal fluid: A cytopathological review of 15 cases. Breast J 23: 456-460, 2017.

13. Bell JE: Update on central nervous system cytopathology. I. Cerebrospinal fluid. J Clin Pathol 47: 573-578, 1994. 
14. Bigner SH: Cerebrospinal fluid (CSF) cytology: Current status and diagnostic applications. J Neuropathol Exp Neurol 51: 235-245, 1992.

15. Cutler RW and Spertell RB: Cerebrospinal fluid: A selective review. Ann Neurol 11: 1-10, 1982

16. Yamada M, Saito A, Yamamoto Y, Cosatto E, Kurata A, Nagao T, Tateishi A and Kuroda M: Quantitative nucleic features are effective for discrimination of intraductal proliferative lesions of the breast. J Pathol Inform 7: 1, 2016.

17. Kosuge N, Saio M, Matsumoto H, Aoyama H, Matsuzaki A and Yoshimi N: Nuclear features of infiltrating urothelial carcinoma are distinguished from low-grade noninvasive papillary urothelial carcinoma by image analysis. Oncol Lett 14: 2715-2722, 2017.

18. EyraudD,GrangerB,Bardier A,LoncarY,Gottrand G,LeNaour G, Siksik JM, Vaillant JC, Klatzmann D, Puybasset L, et al: Immunological environment in colorectal cancer: A computeraided morphometric study of whole slide digital images derived from tissue microarray. Pathology 50: 607-612, 2018

19. Kobayashi S, Saio M, Fukuda T, Kimura K, Hirato J and Oyama T: Image analysis of the nuclear characteristics of emerin protein and the correlation with nuclear grooves and intranuclear cytoplasmic inclusions in lung adenocarcinoma. Oncol Rep 41 : 133-142, 2019.

20. Bosman FT, Carneiro F, Hruban RH and Theise ND (eds.) WHO Classification Of Tumours of the Digestive System. WHO Classification of Tumours, 4th edition, Volume 3. International Agency for Research on Cancer, Lyon, 2010.

21. Louis DN, Ohgaki H, Wiestler OD and Cavenee WK (eds.): WHO Classification Of Tumours of the Central Nervous System. WHO Classification of Tumours, 4th edition, Volume 1. International Agency for Research on Cancer, Lyon, 2007.

22. Travis WD, BrambillaE, Burke AP,Marx A and Nicholson AG (eds.): WHO Classification of Tumours of the Lung, Pleura, Thymus, and Heart. WHO Classification of Tumours, 4th edition, Volume 7. International Agency for Research on Cancer, Lyon, 2015.

23. Swerdlow SH, Campo E, Harris NL, Jaffe ES, Pileri SA, Stein H, Thiele J and Vardiman JW (eds): WHO Classification of Tumours of the Haematopoietic and Lymphoid Tissues. WHO Classification of Tumours, 4th edition, Volume 2. International Agency for Research on Cancer, Lyon, 2008.

24. Douglas CE and Michael FA: On distribution-free multiple comparisons in the one-way analysis of variance. Commun Stat Theory Methods 20: 127-139, 1991.
25. Perkins NJ and Schisterman EF: The inconsistency of 'optimal' cutpoints obtained using two criteria based on the receiver operating characteristic curve. Am J Epidemiol 163: 670-675, 2006.

26. Anand M, Kumar R, Jain P, Asthana S, Deo SV, Shukla NK and Karak A: Comparison of three different staining techniques for intraoperative assessment of nodal metastasis in breast cancer. Diagn Cytopathol 31: 423-426, 2004.

27. Beyer-Boon ME and Voorn-den Hollander MJ: Cell yield obtained with various cytopreparatory techniques for urinary cytology. Acta Cytol 22: 589-593, 1978.

28. Kim J and Bae JS: Tumor-Associated macrophages and neutrophils in tumor microenvironment. Mediators Inflamm 2016: 6058147, 2016.

29. Arenberg DA, Keane MP, DiGiovine B, Kunkel SL, Strom SR, Burdick MD, Iannettoni MD and Strieter RM: Macrophage infiltration in human non-small-cell lung cancer: The role of CC chemokines. Cancer Immunol Immunother 49: 63-70, 2000.

30. Xu H, Zhang Y, Pena MM, Pirisi L and Creek KE: Six 1 promotes colorectal cancer growth and metastasis by stimulating angiogenesis and recruiting tumor-associated macrophages. Carcinogenesis 38: 281-292, 2017.

31. Wang T, Ge Y, Xiao M, Lopez-Coral A, Li L, Roesch A, Huang C, Alexander P, Vogt T, Xu X, et al: SECTM1 produced by tumor cells attracts human monocytes via CD7-mediated activation of the PI3K pathway. J Invest Dermatol 134: 1108-1118, 2014.

32. Chamberlain MC, Kormanik PA and Glantz MJ: A comparison between ventricular and lumbar cerebrospinal fluid cytology in adult patients with leptomeningeal metastases. Neuro Oncol 3: 42-45, 2001.

33. Gaddis GM and Gaddis ML: Introduction to biostatistics: Part 3 , sensitivity, specificity, predictive value, and hypothesis testing. Ann Emerg Med 19: 591-597, 1990.

34. Hazra A and Gogtay N: Biostatistics series module 5: Determining sample size. Indian J Dermatol 61: 496-504, 2016.

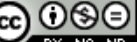

This work is licensed under a Creative Commons Attribution-NonCommercial-NoDerivatives 4.0 International (CC BY-NC-ND 4.0) License. 